\title{
Control of proliferation activation in quiescent neuroblasts of the Drosophila
} central nervous system

\author{
Sumana Datta \\ Department of Biochemistry and Biophysics, Department of Biology, Center for Advanced Invertebrate Molecular Sciences, Texas \\ A\&M University, College Station, Texas, 77843-2128, USA
}

\section{SUMMARY}

Stem cell proliferation is controlled through cell cycle arrest and activation. In the central nervous system of Drosophila melanogaster, neuroblast quiescence and activation takes place in defined spatial and temporal patterns. Two genes have been identified that regulate the pattern of neuroblast quiescence and proliferation. ana, which has been previously described by Ebens and coworkers (Ebens, A., Garren, H., Cheyette, B. N. R. and Zipursky, S. L. (1993). Cell 74, 15-28), encodes a secreted glial glycoprotein that inhibits premature neuroblast proliferation. We previously showed that trol $^{\text {sd }}$ causes a dramatic drop in the number of dividing cells in the larval brain late in development. This study presents evidence that this decrease results from a failure to activate proliferation in the quiescent neuroblast population at the appropriate time. However, trolsd does not affect the maintenance of cell division in already dividing mushroom body neuroblasts. The quiescent optic lobe and thoracic neuroblasts affected by trolsd $^{\text {sd }}$ proliferate in a trol mutant background if they have been activated by a lack of the ana proliferation repressor, demonstrating that trol $^{\text {sd }}$ does not affect cellular viability, nor does trol represent a celltype-specific mitotic factor. This also shows that trol acts downstream of ana to activate proliferation of quiescent neuroblasts in an anadependent pathway, possibly by inactivating or bypassing the ana repressor. These results suggest that trol and ana are components of a novel developmental pathway for the control of cell cycle activation in quiescent neuroblasts.

Key words: Drosophila, central nervous system, stem cell proliferation, neuroblast, ana, trol

\section{INTRODUCTION}

Regulation of cellular proliferation is critical for the development and function of multicellular organisms. The proliferation of specialized progenitor cells provides a pool of cellular precursors for terminally differentiated cell types in many systems. These specialized cells or stem cells are multipotent self-renewing progenitors of terminally differentiated cell types. Stem cells divide symmetrically to increase their population or asymmetrically to regenerate themselves and produce a precursor cell that will form specialized post-mitotic cells. The spatial and temporal pattern of terminally differentiated cell type production is controlled in part by regulation of stem cell proliferation. Quiescent or cell cycle arrested populations of hematopoeitic (Furukawa et al., 1990; Spangrude and Johnson, 1990; Srour et al., 1991; Ogata et al., 1992; Uchida and Weissman, 1992), germline, and nervous system stem cells (Hartenstein and Campos-Ortega, 1984; Campos-Ortega and Hartenstein, 1985; Truman and Bate, 1988; Ito and Hotta, 1991; Prokop and Technau, 1991, 1994; Reynolds and Weiss, 1992; Ebens et al., 1993) have been identified. These stem cells undergo proliferation and quiescence in response to developmental cues. Formally, control of stem cell proliferation can be broken down into (1) activation of proliferation, (2) mainte- nance of the proliferative state, (3) activation of cell cycle repression, and (4) maintenance of cell cycle repression.

To identify components required for cell cycle activation rather than maintenance of cell division, we would like to examine activation of quiescent stem cells in a control background of continually cycling cells. The Drosophila central nervous system (CNS) provides such a system. In the larval brain, three populations of neuroblasts with distinct proliferation profiles have been identified (Fig. 1). Two of these populations undergo cell cycle arrest and activation at specific developmental stages. The third provides a control population of continually dividing cells. The two populations exhibiting specific temporal regulation of proliferation are the optic lobe and thoracic neuroblasts. These neuroblasts begin proliferation during early embryogenesis and become quiescent by late embryogenesis (Hartenstein and Campos-Ortega, 1984; Green et al., 1993). In later larval stages the optic lobe neuroblasts are activated and begin proliferation by late first larval instar or approximately 15 hours posthatching (White and Kankel, 1978; Hofbauer and Campos-Ortega, 1990; Prokop and Technau, 1991; Ebens et al., 1993). The thoracic neuroblasts are activated and begin divisions at 28 hours posthatching (Truman and Bate, 1988). In contrast, the third neuroblast population, the mushroom body neuroblasts, begin dividing during 


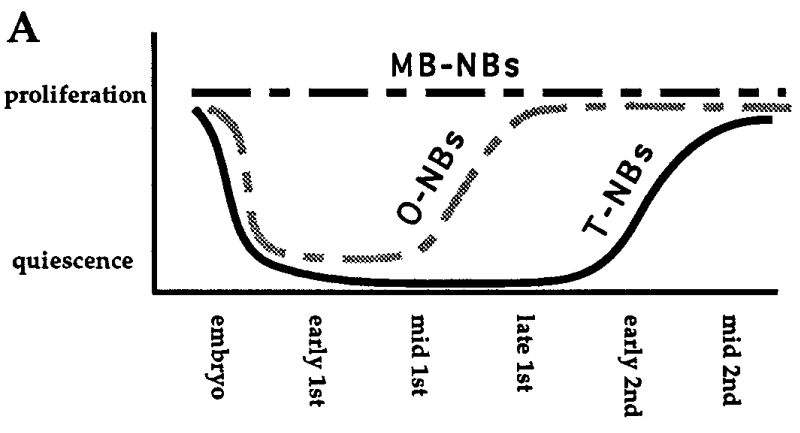

B

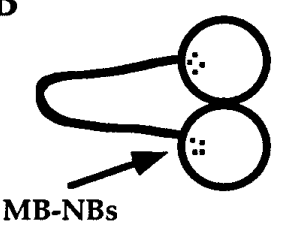

MB-NBs

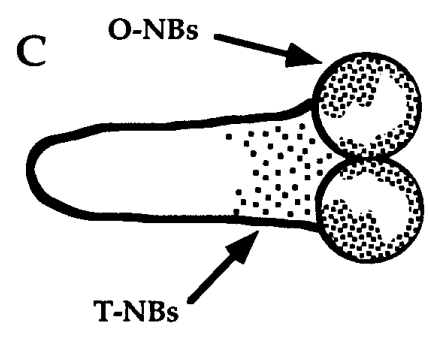

Fig. 1. Developmental proliferation profiles of three neuroblast populations. (A) Qualitative patterns of proliferation and quiescence during embryonic and early larval development. The optic lobe neuroblasts $(\mathrm{O}-\mathrm{NBs})$ proliferate during early embryogenesis, becoming quiescent by 9 hours after egg lay. They remain quiescent until 12-15 hours posthatching, or approximately mid-first instar, when they enlarge and begin proliferation (White and Kankel, 1978; Campos-Ortega and Hartenstein, 1985; Truman and Bate, 1988). The thoracic neuroblasts (T-NBs) proliferate during early embryogenesis, and also become quiescent by late embryogenesis. The thoracic neuroblasts activate and begin cell divisions at about 28 hours posthatching, or early second instar (Truman and Bate, 1988). The mushroom body neuroblasts (MB-NBs) are actively dividing by late embryogenesis and continue proliferating until early pupariation (Ito and Hotta, 1991). (B) Schematic diagram of the spatial pattern of mushroom body neuroblasts (MB-NBs) in the first instar larval brain. Arrow points to the four mushroom body neuroblasts. Panel C. Schematic diagram of the spatial pattern of the optic lobe (O-NBs) and thoracic (T-NBs) neuroblasts in the early second instar larval brain. Labeled arrows indicate the optic lobe neuroblasts in the brain lobes and the thoracic neuroblasts in the ventral ganglion.

Proliferating optic lobe neuroblasts are in the same position in the brain lobes at late first instar. In both B and C anterior is to the left.

embryogenesis and continue to divide until pupariation (Ito and Hotta, 1991). Mutations in genes required specifically for cell cycle activation in the larval stages should affect the optic lobe neuroblasts and thoracic neuroblasts but not the mushroom body neuroblasts. Mutations in genes required for maintenance of mitosis should affect all three populations. Thus the mushroom body neuroblasts can be used as an internal control to eliminate from consideration mutations in genes representing cell cycle components such as cyclin B.

Four Drosophila genes have been identified that affect neuroblast proliferation (Lipshitz and Kankel, 1985; Datta and Kankel, 1992; Ebens et al., 1993; Prokop and Technau, 1994), including anachronism (ana) and terribly reduced optic lobes (trol). trol ${ }^{\text {sd }}$ is a lethal mutation that was initially identified based on aberrant larval brain lobe morphology and small imaginal discs (Datta and Kankel, 1992). Further analysis suggested that rrols $^{s d}$ is a severe partial-loss-of-function allele with a polyphasic lethal period, in that it extends over two larval instars. Mosaic analysis showed that the larval brain defects are not a secondary effect of abnormalities in the compound eye, as has been shown to be the case for mutations at other loci (Power, 1943; Meyerowitz and Kankel, 1978; Selleck and Steller, 1991). The small size of the brain lobe correlates with a severe drop in the number of dividing cells in the lobe and ventral ganglion at early third instar, approximately 50-55 hours posthatching. Surprisingly, the decrease in proliferation in the larval brain appears to be region specific, as there is approximately a $30 \%$ drop in the number of dividing cells in a mutant brain lobe compared to wild-type controls, but a virtually complete lack of proliferation in the thoracic region. This spatial difference in severity of the proliferation defect is apparent in every mutant sample examined $(n=15)$ and suggests that the rol $^{s d}$ mutation might be affecting the proliferation of specific populations of neuroblasts. The lack of proliferating thoracic neuroblasts in trol $^{\text {sd }}$ mutants, for example, suggests that trol is required for thoracic neuroblast proliferation, however the presence of two thirds of the normally dividing cells in the brain lobes suggests that some populations of neuroblasts do not require trol for normal proliferation. In this study, the pattern of neuroblast proliferation in mutant animals early in larval life was examined to determine if particular neuroblast lineages are affected by trol ${ }^{s d}$, or whether the mutation has a general effect on neuroblast proliferation. These studies show that trol is required for initiation of proliferation of a particular subset of neuroblasts.

A second gene, ana, is required for proliferative control of specific neuroblasts (Ebens et al., 1993). ana encodes a secreted glial glycoprotein proposed to repress premature proliferation of quiescent optic lobe and thoracic neuroblasts. However, when proliferation is activated in quiescent neuroblasts, expression of ana-lacZ constructs and levels of ana mutant protein appear constant. This suggests that expression of the ana repressor may remain unchanged during the transition from quiescence to activation of neuroblast proliferation, in which case a second factor must overcome ana repression through bypassing or inactivating the ana repressor to allow proliferation of the optic lobe and thoracic neuroblasts at the appropriate developmental stage. The ana protein shows no significant homology to known proteins and null alleles of ana are viable. No gross structural defects outside the nervous system were observed in animals homozygous for ana null alleles (Datta, unpublished observation).

In this study the proliferation defect caused by the severe hypomorphic allele trol $^{s d}$ has been traced to a failure to activate proliferation of quiescent optic lobe neuroblasts at 16-20 hours posthatching. Maintenance of proliferation by the mushroom body neuroblasts at the same developmental stage remains unaffected in trol $^{\text {sd }}$ mutants suggesting that trol is not required once proliferation has been initiated. Examination of the

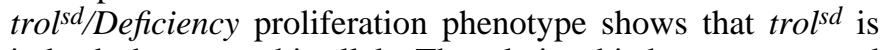
indeed a hypomorphic allele. The relationship between ana and trol has been examined and shows that trol acts downstream of ana, consistent with the possibility that trol may function to inactivate or bypass ana repression to activate proliferation. These results demonstrate that trol is required specifically to regulate initiation of proliferation and may be part of a switch that controls the transition from quiescence to active proliferation. 


\section{MATERIALS AND METHODS}

\section{Genetics}

Markers and balancer chromosomes are described in Lindsley and Zimm (1992). $D f(1) 42$, sn was obtained from and characterized by M. Greenberg. The severe hypomorphic trol $^{\text {sd }}$ allele has been previously described (Datta and Kankel, 1992). trol $^{s d}$ mutant larvae were obtained from the stock $y$ trol $^{\text {sd }}$ w/Binsn. Mutant larvae were unambiguously identified by the larval marker $y$. $\mathrm{rrol}^{\text {sd }} /$ Deficiency samples were obtained as $y$ larvae from $y$ trol $^{\text {sd }}$ w/Binsn females crossed to $y$ $D f(1) 42, s n / w+y+Y$ males. The $a n a^{8}$ null allele was generously provided by Larry Zipursky (Ebens et al., 1993). The trol $^{s d}$; ana ${ }^{8}$ double mutant stock was constructed as $y \mathrm{trol}^{\text {sd }}$ w/Binsn; ana $8 / \mathrm{CyO}$ $y^{+}$or $y$ trol $^{s d}$ w/Binsn; ana $8 / \operatorname{In}(2 L R)$ Gla Elp Bc. This allowed the unambiguous identification of double mutant individuals as $y$ larvae in the former stock or $y ; B c^{+}$larvae in the latter stock.

\section{BrdU labeling and cell counting}

5-bromodeoxyuridine (BrdU) incorporation was done as previously described (Truman and Bate, 1988; Datta and Kankel, 1992). Incorporation was monitored with a primary anti-BrdU mouse antibody (Becton-Dickinson) 1:100 dilution and a goat-anti-mouse secondary coupled to horseradish peroxidase (Gibco) 1:200 dilution. Signal was developed with diaminobenzidine (Truman and Bate, 1988) and samples mounted in $90 \%$ glycerol for viewing on a Zeiss axiophot compound microscope. Labeled neuroblasts were counted visually by examining a continuum of different planes of focus throughout each sample. Proliferating neuroblasts were identified by size and shape of their nucleus when stained for BrdU incorporation. Lateral thoracic neuroblasts were defined as those neuroblasts located in the lateral third of the thoracic portion of the ventral ganglion. Each side of a ventral ganglion was counted and treated as an independent sample. Medial thoracic neuroblasts were not examined. Identification of the mushroom body neuroblasts was accomplished by a BrdU double feeding technique as described by Ito and Hotta (1991). Newly hatched first instar larvae were fed for 3 hours on BrdU containing White/Kankel medium to label the progeny of the mushroom body neuroblasts (the label in the mushroom body neuroblasts themselves becomes diluted out in subsequent divisions), allowed to develop further on normal food, and fed a second time on BrdU-containing medium for 4 hours, at 16 hours posthatching, to label cells that were dividing during that period. The mushroom body neuroblasts were identified both by position and by the presence of a cluster of smaller labeled cells derived from the early labeling period positioned just internally to the presumptive mushroom body neuroblasts. Photomicroscopy was done with Kodak T-max ASA 100 or Kodak Ektapress gold ASA 100 film.

\section{Developmental staging}

Larvae were synchronized by collection of newly hatched first instar larvae in 30- or 60-minute windows. Due to a 2 hour developmental delay in $\mathrm{trol}^{\mathrm{sd}}$ mutant larvae during first instar, mutant samples were labeled from 18-22 hours posthatching and compared to sibling controls that were labeled from 16-20 hours posthatching.

\section{Histological analysis}

Neuroblasts were identified by staining larval brains dissected at 30 hours posthatching with toluidine blue (Ashburner, 1989). Alternatively, larvae were processed for reduced silver staining and sectioned as previously described (Datta and Kankel, 1992).

\section{Statistical analysis}

Standard deviation for each group of samples was calculated and used to determine the confidence limits on the difference between mean cell counts in mutant and control samples using Student's $t$ test.

\section{RESULTS}

The trol $^{s d}$ allele was originally identified on the basis of larval lethality and small, misshapen larval brain lobes. Previous evidence suggested that it might be a severe partial-loss-offunction allele. A null allele of the locus is not currently available. Initial 5-bromodeoxyuridine (BrdU) incorporation studies revealed an almost complete lack of dividing cells in the ventral ganglion and only about one third the normal number of dividing cells in the brain lobes at early third instar or approximately 50 hours posthatching (Datta and Kankel, 1992). However, the mechanism leading to the severe loss of proliferating neuroblasts at this time was unknown. The fact that proliferation of thoracic neuroblasts was rarely seen, but a fair number of brain lobe cells were capable of undergoing mitosis in a trolsd mutant suggested that trol might be required for the activation or maintenance of proliferation of specific lineages such as the thoracic neuroblast lineage. One possibility was that trol $^{\text {sd }}$ mutants might affect the proliferation of specific neuroblasts. If so, those lineages should be missing in the mutant. A second possibility was that trol $^{s d}$ mutants affect cell fitness, which would be more apparent in rapidly growing cell populations. In this case a relatively uniform pattern of defective proliferation affecting all types of neuroblast populations irrespective of their pattern of proliferation regulation should be apparent in the mutant. To distinguish between these possibilities, and examine the origin of the aberrant proliferation pattern in the larval brain, BrdU analysis was performed at early larval stages to characterize the first visible proliferation defects.

\section{Quiescent optic lobe neuroblasts fail to begin proliferation in trolsd}

The entry of the optic lobe neuroblasts into $\mathrm{S}$ phase was monitored by BrdU incorporation in vivo at $16-20$ hours posthatching. Larval brains were dissected with mouth hooks attached for ease of handling, and processed to identify cells that had incorporated BrdU into their DNA during the labeling period. Counts of labeled neuroblasts were made in each hemisphere of the larval brain, with the majority of neuroblasts appearing in the optic lobe proliferation zones (Fig. 2). All cells that have passed through $\mathrm{S}$ phase during the labeling period incorporate $\mathrm{BrdU}$, including ganglion mother cells, immature neurons, and neuroblasts. Neuroblasts were identified by the large size of their nuclei relative to other dividing cells and the somewhat irregular shape of the nucleus. Examples of physical differences between labeled neuroblasts and the smaller ganglion mother cells and immature neurons are shown in Fig. 2. A 4-hour BrdU incorporation in wild-type larvae from 16-20 hours posthatching resulted in the labeling of $19.9 \pm 1.2$ neuroblasts per brain lobe $(n=8)$. However, a 4hour labeling of trol $^{\text {sd }}$ hemizygous larvae from 18-22 hours posthatching resulted in the labeling of $11.7 \pm 1.6$ neuroblasts per brain lobe $(n=14)$ (Table 1). The shift in labeling period for the mutant samples was used to correct for a developmental delay in mutant animals. trol mutant animals take an extra 2 hours to develop from hatching to a well defined morphological transition at first to second larval instar. Normally this period of larval life is 24 hours long, but in trol mutant animals this developmental period takes 26 hours (Datta, unpublished observations). Since it is unknown whether the extra 2 hours 


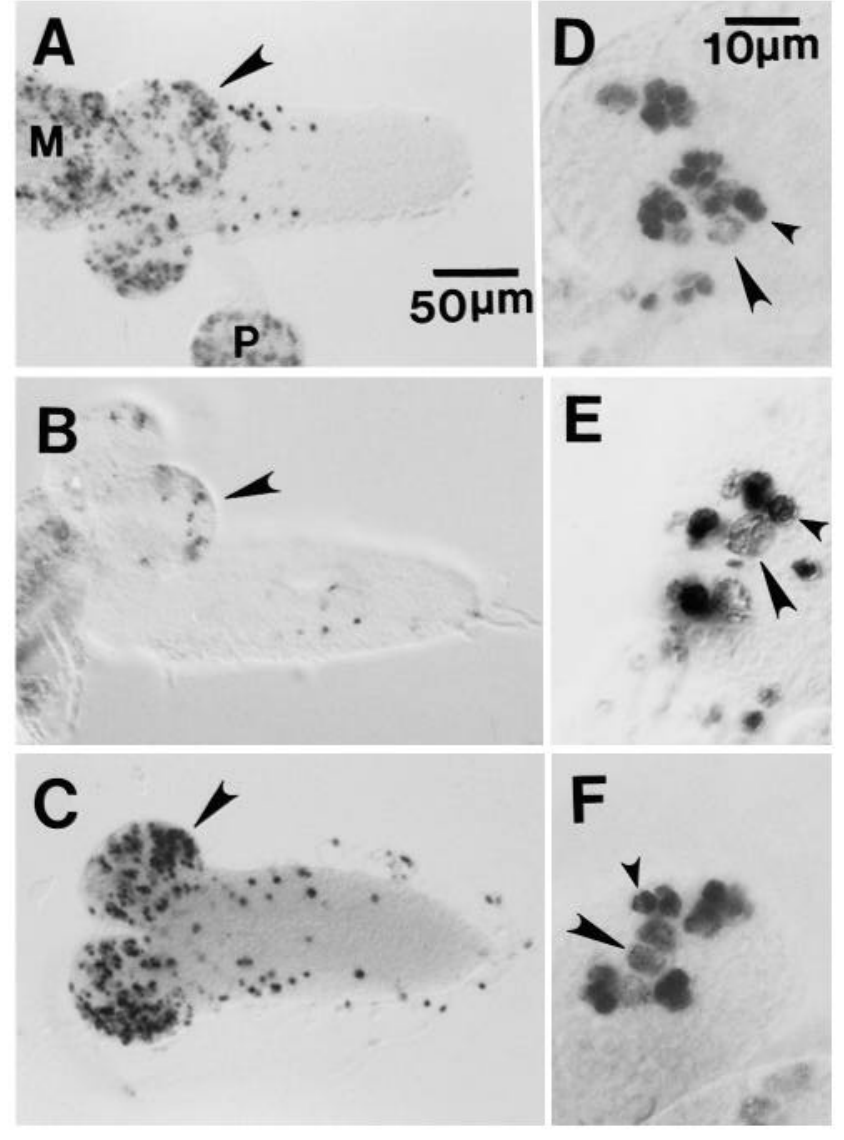

Fig. 2. Proliferation defect in late first instar. The number of proliferating neuroblasts per brain lobe was assayed in rrol $^{\text {sd }}$ mutant, $\mathrm{trol}^{s d} / \mathrm{Df}(1) 42$, and control brain lobes by incorporation of BrdU. Each set of samples was collected within a 1 hour window to synchronize development. Mutant samples were labeled from 18-22 hours posthatching to correct for developmental delay, control samples were labeled from 16-20 hours posthatching. Representative sample of (A) trolsd mutant brain with attached mouthhooks (M) and proventriculus (P), (B) trolsd/Df(1)42 brain, and (C) control brain at $20 \times$ magnification. Arrowhead indicates brain lobe. Bar indicates 50 $\mu \mathrm{m}$. Maintenance of proliferation by the mushroom body neuroblast as assayed by BrdU incorporation. Representative sample of (D) 0-4 hour trolsd, (E) 18-22 hour trolsd/Df(1)42, and (F) 0-4 hour control brain lobes at $80 \times$ magnification. Large arrowheads indicate a mushroom body neuroblast, small arrowheads indicate a ganglion mother cell. Bar indicates $10 \mu \mathrm{m}$. In all panels anterior is to the left.

are required for trol mutant animals soon after hatching, spread over the entire first developmental stage, or at the end of the usual 24 hour period, labeling times for mutant animals were offset from control feedings by the entire 2 hour period. This 2 hour offset at 16 hours is a conservative correction in that it assumes that all of the extra 2 hours of developmental time is required prior to the stage represented by 16-hour posthatching normal animals. Thus trol mutant animals at 18 hours posthatching will be either at the same developmental stage as 16-hour posthatching controls, or at a slightly later developmental stage when more neuroblasts should be actively dividing. Thus, the deficit in the number of dividing neuroblasts in the mutant revealed by this analysis is probably an underestimate of the magnitude of the defect. The reduction in the number of dividing neuroblasts represents a significant $(P<<0.05)$ drop of forty percent in trol $^{\text {sd }}$ mutant brains at a time when the majority of proliferating neuroblasts are previously quiescent cells that have recently started dividing. This suggests that the proliferation defect exhibited by trol $^{\text {sd }}$ mutant animals may be due to an inability to activate proliferation in quiescent neuroblasts.

\section{Maintenance of mushroom body neuroblast proliferation is unaffected in tro/sd}

To determine if the drop in the number of proliferating neuroblasts was due to a general neuroblast proliferation defect at 16-22 hours posthatching or a specific defect in activation of quiescent neuroblasts, the proliferation of a class of continuously dividing neuroblasts was examined as a control population. The proliferation of the mushroom body neuroblasts was monitored in mutant and control animals. The early proliferation of the four mushroom body neuroblasts and the single lateral neuroblast was assayed at 4 hours posthatching by BrdU incorporation (Fig. 2). At this stage, the mushroom body and lateral neuroblasts are the only dividing neuroblasts in the larval CNS (Truman and Bate, 1988; Ito and Hotta, 1991). All five of the neuroblasts were labeled in every mutant $(n=28)$ and control sample $(n=10)$ (Table 1), indicating that normal levels of trol are not required for the maintenance of neuroblast proliferation at 0-4 hours posthatching.

Although mushroom body neuroblast proliferation seemed unaffected by trol ${ }^{s d}$ at $0-4$ hours posthatching, it was possible that proliferation of all neuroblasts is normal in trol mutants very early in larval life and is only disrupted later when reduction in the number of dividing neuroblasts becomes apparent. If this is true, then maintenance of proliferation of the mushroom body neuroblasts at later times should be affected. Continuing division of the mushroom body neuroblasts at 16-22 hours posthatching was addressed in a second experiment. The larger number of dividing neuroblasts at this stage makes it more difficult to identify the mushroom body neuroblasts after a single BrdU labeling. Thus the double feeding technique of Ito and Hotta (1991) was used to identify the mushroom body neuroblasts at 16-22 hours posthatching. This procedure labels the progeny of the mushroom body neuroblasts in newly hatched larvae with a pulse of BrdU, after which the larvae continue development on normal medium. The larvae are labeled a second time with BrdU at 16-20 hours posthatching for control and 18-22 hours posthatching for mutant animals. The putative mushroom body neuroblasts were identified by position and the presence of a cluster of small labeled cells just internal to the neuroblast. Since there is very little cell migration in the Drosophila brain during larval life, the small cells represent the previous progeny of the mushroom body neuroblasts labeled during the first BrdU pulse, which remain closely associated with the mushroom body neuroblasts. This study showed that all the mushroom body neuroblasts were dividing in both control (16-20 hour labeling, 3.7 \pm 0.6 mushroom body neuroblasts, $n=12)$ and mutant (18-22 hour labeling, $3.9 \pm 0.3$ mushroom body neuroblasts, $n=14$ ) animals at 16-22 hours posthatching (Fig. 2 and Table 1). Taken together, these results imply that normal levels of trol are not required for maintenance of mushroom body neuroblast proliferation at early or late first instar. 
Table 1. Summary of brain lobe and mushroom body neuroblast proliferation

\begin{tabular}{lccc}
\hline & $\begin{array}{c}\text { Brain lobe } \\
\text { late labeling }\end{array}$ & $\begin{array}{c}\text { Mushroom body } \\
\text { early labeling }\end{array}$ & $\begin{array}{c}\text { Mushroom body } \\
\text { late labeling }\end{array}$ \\
\hline trol $^{\text {sd }}$ & $11.7 \pm 1.6$ & 4.0 & $3.9 \pm 0.3$ \\
& $n=14$ & $n=28$ & $n=14$ \\
trol $^{\text {sd } / D f}$ & $6.7 \pm 0.8$ & n.d. & $3.8 \pm 0.3$ \\
& $n=13$ & & $n=13$ \\
normal & $19.9 \pm 1.2$ & 4.0 & $3.7 \pm 0.6$ \\
& $n=8$ & $n=10$ & $n=12$
\end{tabular}

Late labeling periods were 16-20 hours posthatching for control and 18-22 hours posthatching for rrol $^{\text {sd }}$ mutant samples. Early labeling period was 0-4 hours posthatching for both samples. n.d. indicates not done.

\section{tro/sd/Deficiency larvae have a more severe neuroblast proliferation phenotype}

Since trol ${ }^{s d}$ is suspected to be a hypomorphic allele, the phenotype of trolsd/Deficiency animals was examined to determine whether further reduction in the level of trol would result in abnormal mushroom body neuroblast proliferation. A 4-hour BrdU incorporation in mutant/Deficiency animals $(n=13)$ from 18-22 hours posthatching (Fig. 2) resulted in the labeling of $6.7 \pm 0.8$ neuroblasts per brain lobe compared with $11.7 \pm 1.6$ in trol $^{\text {sd }}$ mutants. However, further examination revealed that despite the further drop in the number of dividing brain lobe neuroblasts in the trolsd/Deficiency animals, virtually every mushroom body neuroblast $(3.8 \pm 0.3, n=13)$ was still dividing (Table 1). This further supports the hypothesis that trol is required for activation of proliferation in quiescent neuroblasts.

\section{Thoracic neuroblasts are still present in mutant larvae}

To determine if quiescent neuroblasts fail to begin proliferation in trol $^{s d}$ mutants because trol is required for viability, the presence or absence of thoracic neuroblasts was assayed at 30 hours posthatching. At this stage thoracic neuroblasts, which are dividing in control animals, fail to incorporate BrdU in trolsd $^{s d}$ mutants (data not shown). Whole-mount toluidine blue staining showed large superficially placed cells in the ventral ganglion (data not shown). Sections through trol ${ }^{\text {sd }}$ mutant samples revealed numerous large superficially placed cells with large nuclei on the ventral aspect of the CNS (Fig. 3). These data indicate that thoracic neuroblasts are still present in mutant larvae although they are not mitotically active.

\section{The trol ; ana double mutant has a premature proliferation phenotype}

Animals mutant for rrols $^{s d}$ appear to be unable to activate proliferation of quiescent neuroblasts at the appropriate developmental stage. The proliferation of quiescent neuroblasts is also affected by mutations at the ana locus, which result in premature proliferation of the optic lobe and thoracic neuroblast populations (Ebens et al., 1993). To determine the epistatic relationship between ana and trol, the proliferation phenotype of a trol ${ }^{\text {sd }}$; ana $^{8}$ double mutant was examined. BrdU labeling experiments at 18-22 hours posthatching demonstrated that the rrol $^{s d} ; a n a^{8}$ double mutant has a proliferation phenotype indistinguishable from the $a n a^{8}$ homozygous animal. Double mutant animals show a large increase in the number of dividing neuroblasts similar to that seen in $a n a^{8}$ mutants (Fig. 4). Quantitation of the number of proliferating lateral thoracic neuroblasts (Table 2) in $\mathrm{trol}^{\mathrm{sd}}$; ana $\mathrm{a}^{8}$ double mutants $(24 \pm 5, n=6)$ compared to $a n a^{8}$ mutants $(21 \pm 2, n=7)$, trol $^{\text {sd }}$ mutants $(0.1 \pm 0.4, n=14)$ and normal samples $(1.1 \pm 0.3$, $n=8)$ demonstrated that the thoracic neuroblast phenotype of the double mutant was indistinguishable from that of the $a n a^{8}$ mutant $(P<<0.005)$, but significantly different from both the trol $^{s d}$ mutant phenotype and the normal pattern of thoracic neuroblast proliferation $(P<<0.005)$. The proliferation of numerous thoracic neuroblasts in the double mutant animals is particularly striking in view of the fact that this does not normally occur until 28 hours posthatching (Truman and Bate, 1988), a good 6 to 8 hours later, and does not appear to occur
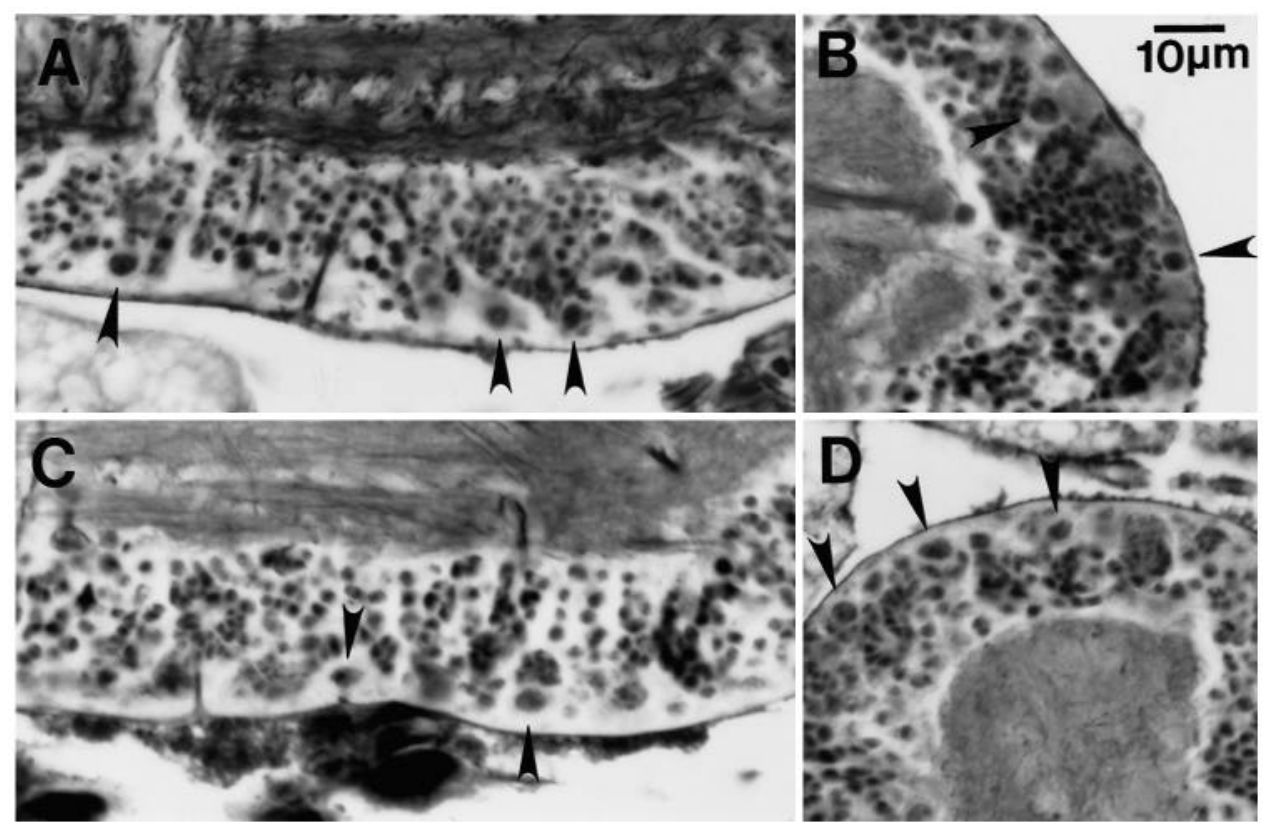

Fig. 3. Presence of thoracic neuroblasts. Sections through a 30 hour posthatching trols $^{s d}$ mutant (A) ventral ganglion and (B) brain lobe, and a control (C) ventral ganglion and (D) brain lobe at $80 \times$ magnification. Arrowheads indicate neuroblasts. Anterior is to the right, bar indicates $10 \mu \mathrm{m}$. 


\section{S. Datta}

in trol $^{\text {sd }}$ mutants even by 50 hours posthatching (Datta and Kankel, 1992).

\section{Other defects are not rescued by ana in the double mutant}

Examination of the double mutant stock revealed that trol ${ }^{\text {sd }}$; an $a^{8}$ individuals do not survive to adulthood. Although the phase of lethality has not been systematically identified, failure to observe dead pupae or double mutant climbing third instar larvae coupled with the identification of viable second instar double mutant individuals suggest that the majority of lethality occurs between early second and late third larval instar or 30-90 hours posthatching. This does not eliminate the possibility that the double mutant combination exhibits polyphasic lethality similar to the trol mutant phenotype.

\section{DISCUSSION}

Many studies have linked the expression of growth factors, hormones, and their receptors with stem cell proliferation. However, studies of unmarked populations do not

Fig. 4. Double mutant proliferation phenotype in the larval brain. Neuroblast proliferation was monitored by incorporation of BrdU. trolsd $; a n a^{8}$ and trol $^{\text {sd }}$ samples were labeled from 18-22 hours posthatching, $a n a^{8}$ and normal samples were labeled from 16-20 hours posthatching (see text for discussion of offset). Samples were collected in a 1-hour window to synchronize development. (A) Lobe of a rrol $^{\text {sd }}$; ana $a^{8}$ double mutant brain showing a large number of dividing neuroblasts. (B) Thoracic region of a trol ${ }^{\text {sd }}$ ; $a n a^{8}$ double mutant brain showing premature proliferation of thoracic neuroblasts. (C) Lobe of an $a n a^{8}$ mutant brain exhibiting a large number of proliferating neuroblasts. (D) Thoracic region of an $a n a^{8}$ mutant brain exhibiting premature proliferation of the thoracic neuroblasts. (E) Lobe of a trol $^{\text {sd }}$ mutant brain showing a reduced number of dividing neuroblasts. (F) Thoracic region of a trol $^{\text {sd }}$ mutant brain showing no proliferating thoracic neuroblasts.

(G) Lobe of a normal brain exhibiting some proliferating neuroblasts.

(H) Thoracic region of a normal brain showing one dividing thoracic neuroblast. In all panels anterior is up, samples are shown at $80 \times$ magnification. Arrowheads point to labeled neuroblasts. Bar represents $10 \mu \mathrm{m}$. allow for the distinction between activation of proliferation and maintenance of proliferation or survival. Epidermal growth factor (EGF) is capable of stimulating thymidine incorporation in vitro in a number of primary neuronal cell culture systems (DiCicco-Bloom et al., 1990), leading to the attribution of mitogenic activity to EGF. Yet recent studies showed that
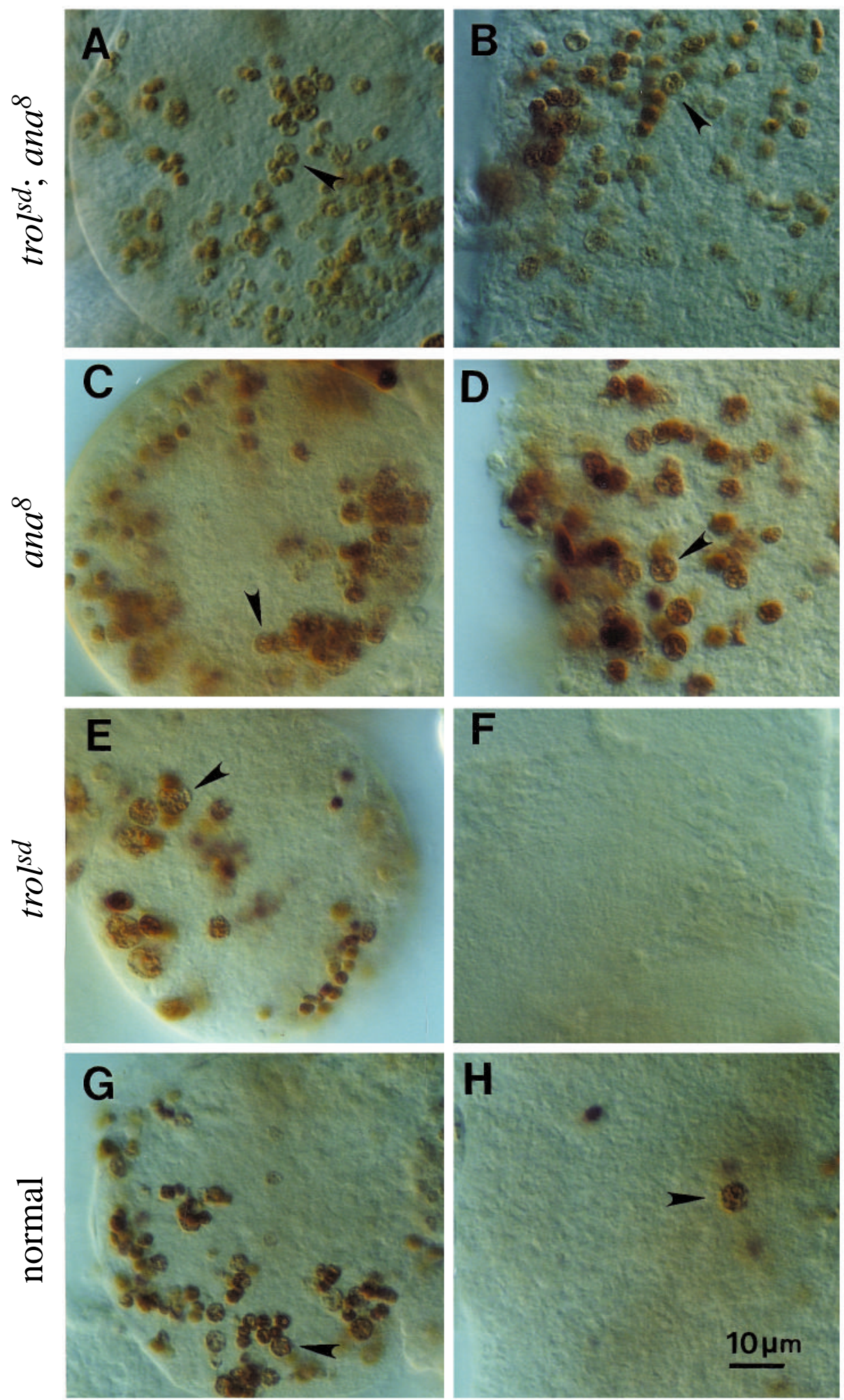
Table 2. Summary of thoracic neuroblast proliferation

\begin{tabular}{lccc}
\hline trol $^{\text {sd }} ;$ ana $^{8}$ & ana $^{8}$ & trol $^{\text {sd }}$ & Normal \\
\hline $24 \pm 5$ & $21 \pm 2$ & $0.1 \pm 0.4$ & $1.1 \pm 0.3$ \\
$n=6$ & $n=9$ & $n=14$ & $n=8$
\end{tabular}

trol $^{s d} ;$ and $^{8}$ and trol $^{\text {sd }}$ mutant samples were labeled from 18-22 hours posthatching.

$a n a^{8}$ and normal samples were labeled from 16-20 hours posthatching.

removal of EGF from a mouse embryonic or adult striatal culture results in a cessation of cell division (Reynolds et al., 1992; Reynolds and Weiss, 1992), suggesting that the presence of EGF may be required to maintain proliferation rather than to initiate cell cycle activation in quiescent cells.

\section{trol is required for activation but not maintenance of proliferation}

Examination of proliferation patterns in $\mathrm{trol}^{s d}$ mutants shows that only specific populations of neuroblasts are affected. trol $^{\text {sd }}$ mutants exhibit a $40 \%$ drop in the number of dividing neuroblasts at 18-22 hours posthatching when previously quiescent optic lobe and central brain neuroblasts normally begin proliferation. In contrast, normal mushroom body neuroblast proliferation patterns are observed (Fig. 2 and Table 1). In every 0-4 hour posthatching sample examined, the four mushroom body neuroblasts and the lateral neuroblast were labeled with BrdU. The decrease in the number of dividing neuroblasts by $18-22$ hours posthatching is not due to arrest of the previously dividing mushroom body neuroblasts, as the failure of four neuroblasts to continue division would be insufficient to explain a decrease of approximately eight dividing neuroblasts per brain lobe in trols ${ }^{s d}$ mutants. Additionally, double labeling experiments show that the mushroom body neuroblasts continue to divide at $16-20$ hours posthatching.

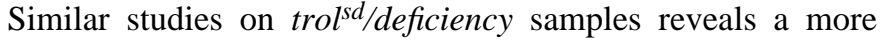
severe $65 \%$ drop in the total number of dividing brain lobe neuroblasts, yet again the proliferation of the mushroom body neuroblasts is unaffected (Fig. 2 and Table 1). This strongly suggests that trol $^{\text {sd }}$ does not result in a general proliferation defect at 16-22 hours posthatching. The drop in the number of dividing neuroblasts must be due to a failure to activate quiescent optic lobe and central brain neuroblasts that begin to proliferate at this stage in wild-type individuals. Mutant brains from later in larval development show vacuolization of the regions that should contain the optic lobe and thoracic neuroblasts (Datta and Kankel, 1992), suggesting that the inability to activate proliferation in a timely fashion leads to eventual cell death. Whether this cell death is apoptotic or necrotic has not been examined.

It has been suggested that quiescent neuroblasts are arrested in G1 of the cell cycle. Since most embryonic cells arrest in G1 (Edgar and O'Farrell, 1990), postembryonic neuroblasts must undergo DNA synthesis before entering mitosis (Truman and Bate, 1988). In addition, late third instar optic lobe neuronal precursors at 75-96 hours posthatching have been shown to arrest in G1 prior to contact from ingrowing retinular axons (Selleck and Steller, 1991; Selleck et al., 1992). This implies S phase is the first cell cycle phase for newly activated neuroblasts. The optic lobe neuroblast cell cycle has been estimated to last approximately 8-9 hours (Hofbauer and
Campos-Ortega, 1990). Since the labeling period used in these studies is at most half of the expected cell cycle period at the time of the first expected cell cycle, it is highly unlikely that the decrease in the number of labeled neuroblasts is due to cells that undergo one round of mitosis but cannot maintain sufficient metabolic activity to continue. In addition, since DNA synthesis precedes the first cell division, the severe decrease in the number of neuroblasts entering $\mathrm{S}$ phase, as detected by BrdU incorporation, reflects an inability of the unlabeled neuroblasts to make the G1-S transition rather than initiation of an elongated cell cycle in trol $^{\text {sd }}$ mutants. Coupled with the observation that the mushroom body neuroblasts, which were dividing at 0-4 hours posthatching, continue to divide at 16-20 hours posthatching, these results suggest that trol is required for activation of proliferation in quiescent optic lobe and central brain neuroblasts but not for maintenance of proliferation in actively dividing mushroom body neuroblasts.

\section{Repression of premature proliferation occurs prior to trol-induced activation}

The regulation of quiescent neuroblast proliferation is also affected by mutations at the ana locus (Ebens et al., 1993). ana encodes a secreted glial glycoprotein with no significant homology to any known proteins. ana expression is first visible in the embryonic central nervous system at stage 14, and continues to be expressed throughout larval life. In ana null mutants, optic lobe neuroblasts enter S phase 8 hours early, suggesting that these cells become competent to divide 8 hours before they begin proliferation. It has been proposed that ana represses premature proliferation of division-competent neuroblasts. When quiescent neuroblasts are activated, however, no change in the levels of ana expression is detected, as monitored by expression of ana-lac $Z$ fusions and levels of mutant protein. The author's observation that levels of ana transcription and translation apparently remain unchanged at the time when the optic lobe neuroblasts initiate cell division suggests that another factor bypasses ana repression of neuroblast activation or directly inactivates the ana repressor pathway. The predicted phenotype of a mutation in such a factor is the continued repression of optic lobe and thoracic neuroblast proliferation by the ana repressor, a phenotype similar to the trol ${ }^{s d}$ mutant phenotype. The trolsd mutant phenotype is also consistent with a function of trol in rendering neuroblasts competent to enter the proliferative phase. The latter would predict that trol is epistatic to ana, the former that ana is epistatic to trol.

The trol ${ }^{s d} ;$ ana $^{8}$ double mutant exhibits premature optic lobe and thoracic neuroblast proliferation (Fig. 4). Examination of thoracic neuroblast proliferation has shown that the extent of proliferation of this population in the $\mathrm{trol}^{\mathrm{sd}} ; \mathrm{ana}^{8}$ double mutant is indistinguishable from the proliferation of thoracic neuroblasts in $a n a^{8}$ mutants at the same developmental stage (Table 2). The observation of numerous dividing thoracic neuroblasts in the double mutant at 22 hours posthatching, 6-8 hours before such activation would normally be seen in wild-type individuals and at a level that is never seen in trol mutant individuals at any time thus far examined, clearly shows that the double mutant exhibits a premature neuroblast proliferation or ana mutant phenotype. According to the classic definition of epistasis, the fact that the trolsd $^{\text {; }}$; ana $a^{8}$ double mutant shows an $a n a^{8}$ mutant phenotype demonstrates that ana 

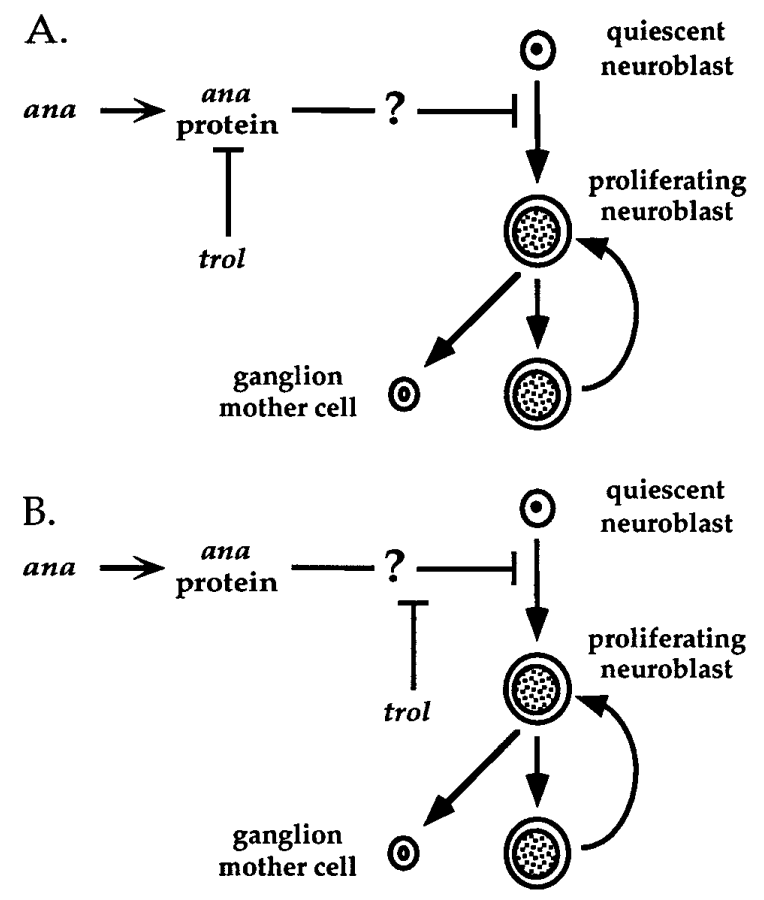

Fig. 5. Models for the control of neuroblast proliferation. ana represses the transition from quiescence to active cell division. A shows how trol may activate neuroblast proliferation by directly inactivating the ana repressor. B shows trol activating neuroblast proliferation by bypassing ana repression downstream in the ana pathway.

is epistatic to trol. In developmental terms, this indicates that ana function must occur prior to the requirement for trol. Thus, if ana represses premature proliferation and trol acts downstream of ana to bypass or inactivate the ana repressor (see Fig. 5 for possible models), elimination of both ana and trol activities as in the double mutant should lead to an ana mutant phenotype, since without ana function there is no consequence of not having trol present to inactivate or bypass the repressor.

This argument implies that if trol acts to bypass the anainduced proliferation blockage, initiating the proliferative cycle further downstream, then this initiating trol activity is required only when events further upstream are blocked by the ana repressor, and is not required in the absence of the repressor. Otherwise, if trol represented a downstream anaindependent activation signal, in the double mutant the lack of that signal would lead to a trol mutant phenotype. Elimination of an independent induction activity encoded by trol in the double mutant would allow neuroblasts to continue through the point at which ana normally represses neuroblast proliferation, but cause them to mitotically arrest where trol would normally induce progression to the next cell cycle stage, resulting in a deficit in the number of dividing neuroblasts in the double mutant rather than the observed premature proliferation phenotype. Therefore, trol should be part of an ana-dependent pathway for glial regulation of quiescent neuroblast proliferation repression and activation. Interactions between glia and neuroblasts that affect neuroblast proliferation have been described in mammalian systems. Murine studies have implicated glia in the repression of granule cell neuroblast prolifer- ation (Gao et al., 1991), although the specific molecules involved in the interaction have yet to be identified.

\section{Implications for the role of trol in regulating neuroblast proliferation}

In the double mutant, the optic lobe and thoracic neuroblasts that did not proliferate in trol $^{s d}$ mutant larvae are capable of mitosis, and in fact, begin proliferation too soon. In addition, thoracic neuroblasts that do not divide in trolsd mutants are visible in stained sections of mutant ventral ganglia (Fig. 3). These observations have several important implications for the nature of trol gene function: (1) trol does not encode a celltypespecific mitotic component, (2) trol ${ }^{\text {sd }}$ does not cause the initial lack of optic lobe and thoracic neuroblast proliferation through death of the affected populations, and (3) trol $^{\text {sd }}$ does not affect cell fitness to the point of rendering the neuroblasts incapable of beginning cell divisions. Regardless of whether trol function is required upstream or downstream of the ana repressor, if trol did function as described in any of the above three possibilities, the double mutant combination would have exhibited a trol mutant phenotype since the optic lobe and thoracic neuroblasts would be incapable of initiating mitosis regardless of the lack of the ana repressor.

This result in combination with the proliferation phenotype

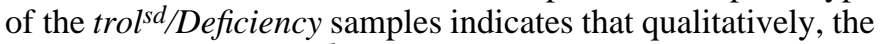
phenotype of the trol ${ }^{s d}$ allele is not due to the hypomorphic nature of the mutation. Presumably, optic lobe and thoracic neuroblasts have the same levels of trol gene product in the trol $^{\text {sd }}$; ana $^{8}$ double mutant as they do in the trolsd single mutant. I have shown that these cells are capable of mitosis in the double mutant. This suggests that the lack of optic lobe and thoracic neuroblast cell division in rrol $^{\text {sd }}$ mutants is not due to a requirement for higher levels of trol gene product for activation of proliferation and lower levels for maintenance of proliferation. In addition, further lowering the levels of trol using trol $^{\text {sd }} /$ Deficiency animals results in a dramatic $65 \%$ drop in the number of proliferating brain lobe neuroblasts, but leaves the continually dividing mushroom body neuroblasts unaffected. This raises the possibility that a null allele might result in a complete lack of quiescent neuroblast activation, leaving only the already dividing neuroblasts capable of proliferating. These results suggest that the central nervous system phenotype of the partial-loss-of-function allele may qualitatively, but not quantitatively, mirror the phenotype of a null allele.

Previous studies have shown that trol may have a maternal and early zygotic function (Garcia-Bellido and Robbins, 1983; Robbins, 1983). If trol also affects nervous system development in embryogenesis, it is possible that maternal contribution may rescue aberrant embryonic neuroblast proliferation patterns in mutant animals.

\section{trol may be part of a general proliferative control mechanism}

Null mutations of ana are viable, and yet the trol $^{\text {sd }} ; a n a^{8}$ double mutant is lethal. If all aspects of the trolsd mutant phenotype were the result of lack of trol function downstream of ana, the double mutant should be viable. This suggests that trol also plays a role in pathways where ana function is either not present or redundant. The mutant phenotype of the trolsd allele includes small imaginal discs (Datta and Kankel, 1992), and mosaic studies documenting effects of other trol alleles on 
wing and leg development have been previously reported (Shannon et al., 1972). trol may be required for ana-independent proliferative control outside the central nervous system. Alternatively, if ana homologs are expressed in imaginal discs, there may be a independent mechanism for the repression of proliferation in these tissues that is still functional in an $a n a^{8}$ mutant background.

trol mutations also affect other developmental processes. Oogenesis has been examined in viable trol homozygotes and trans-heterozygotes. These females produced few or no eggs but developed many stage 14 oocytes (Shannon et al., 1972). Generation of germline clones of a different trol allele resulted in normal numbers of eggs that either could not be fertilized or were incapable of development (Garcia-Bellido and Robbins, 1983). By analogy to trol action in the developing central nervous system, one might expect trol to be required for activation of germline stem cell divisions. However, recent ablation studies have suggested that lack of dividing germline stem cells would lead to ovaries empty of developing egg chambers (Lin and Spradling, 1993). This is clearly not the phenotype reported for trol, although those phenotypes were arrived at by analysis of hypomorphic alleles of varying severity or phenotypes implied from variegating duplications. A clear definition of the effect of trol on oogenesis will require clonal analysis using a null allele. However, it is intriguing to note that the regulation of germline stem cell division has been traced in part to interactions between somatic cells and germline stem cells in both Drosophila (Lin and Spradling, 1993) and C. elegans (Kimble and White, 1981), reminiscent of the interaction between glia and neuroblasts during brain development.

Regulation of the spatial and temporal pattern of neuroblast proliferation in the developing brain is accomplished at least in part by cell-cell interactions between neuroblasts and surrounding glial cells and signal transduction mechanisms that operate within the neuroblast itself. These studies show that the product of the trol locus is part of the developmental switch required for the quiescent-proliferative transition. trol acts downstream of ana, a known repressor of neuroblast proliferation, and may function to bypass the block imposed by the ana protein. This is not meant to imply that trol is downstream of ana in the context of a direct cascade of transcriptional events within the neuroblast. It is certainly possible that trol represents a second intercellular signal that has a competing inductive function on neuroblast proliferation rather than a repressive function. Alternatively, trol could interfere with glial secretion of the ana repressor although the effect of trol mutations on proliferating systems not affected by mutations at ana make this hypothesis somewhat unlikely. Further molecular genetic analysis of the trol-ana functional relationship is required to determine if trol and ana interact directly in a signal transduction mechanism. trol and ana represent a pathway through which developmental cues can be transduced to control cell cycle activation in the developing brain in a specific spatial and temporal pattern. The observation that mutations in trol affect other developing tissues, and that the trolsd $^{\text {sd }}$; ana $a^{8}$ double mutant is lethal suggest that trol may be part of a more general mechanism for the developmental control of cellular proliferation. Further molecular and cellular characterization of trol and its interaction with ana are necessary to understand the molecular mechanisms through which these developmental signals interface with cell cycle machinery.

I would like to thank Drs Larry Zipursky and Allen Ebens for the ana fly stocks, and Dr Mitzi Kuroda for the $\mathrm{CyO} y^{+}$balancer. I would also like to thank Doug Dimlich and Drs Marianne Bronner-Fraser, Craig Woodard, and Allen Ebens for critical reading of the manuscript and Doug Dimlich, and Drs Marianne Bronner-Fraser, Doug Kankel, Haig Keshishian, and Kalpana White for helpful discussions. Technical support was provided by Sean Hill and Casey Hefflin. This work was supported by a Grant-in-Aid from the American Heart Association, Texas Affiliate, Inc. to S. D. S. D. is an American Cancer Society Junior Faculty Fellow.

\section{REFERENCES}

Ashburner, M. (1989). Drosophila: A Laboratory Manual. Cold Spring Harbor, New York: Cold Spring Harbor Laboratory Press.

Campos-Ortega, J. A. and Hartenstein, V. (1985). The Embryonic Development of Drosophila melanogaster. Berlin: Springer-Verlag.

Datta, S. and Kankel, D. R. (1992). l(1)trol and l(1)devl, loci affecting the development of the adult central nervous system in Drosophila melanogaster. Genetics 130, 523-537.

DiCicco-Bloom, E., Townes-Anderson, E. and Black, I. B. (1990). Neuroblast mitosis in dissociated culture: regulation and relationship to differentiation. J. Cell Biol. 110, 2073-2086.

Ebens, A. J., Garren, H., Cheyette, B. N. R. and Zipursky, S. L. (1993). The Drosophila anachronism locus: A glycoprotein secreted by glia inhibits neuroblast proliferation. Cell 74, 15-28.

Edgar, B. A. and O'Farrell, P. H. (1990). The three postblastodermal cell cycles of Drosophila embryogenesis are regulated in $\mathrm{G} 2$ by string. Cell 62, 469-480.

Furukawa, Y., DeCaprio, J. A., Freedman, A., Kanakura, Y., Nakamura, M., Ernst, T. J., Livingston, D. M. and Griffin, J. D. (1990). Expression and state of phosphorylation of the retinoblastoma susceptibility gene product in cycling and noncycling human hematopoietic cells. Proc. Natl. Acad. Sci. USA 87, 2770-2774.

Gao, W.-Q., Heintz, N. and Hatten, M. E. (1991). Cerebellar granule cell neurogenesis is regulated by cell-cell interactions in vitro. Neuron $\mathbf{6}, 705$ 715 .

Garcia-Bellido, A. and Robbins, L. G. (1983). Viability of female germ-line cells homozygous for zygotic lethals in Drosophila melanogaster. Genetics 103, 235-247.

Green, P., Hartenstein, A. Y. and Hartenstein, V. (1993). The embryonic development of the Drosophila visual system. Cell Tissue Res. 273, 583-598.

Hartenstein, V. and Campos-Ortega, J. A. (1984). Early neurogenesis in wild-type Drosophila melanogaster. Roux's Arch. Dev. Biol. 193, 308-325.

Hofbauer, A. and Campos-Ortega, J. A. (1990). Proliferation pattern and early differentiation of the optic lobes in Drosophila melanogaster. Roux's Arch. Dev. Biol. 198, 264-274.

Ito, K. and Hotta, Y. (1991). Proliferation pattern of postembryonic neuroblasts in the brain of Drosophila melanogaster. Dev. Biol. 149, 134148 .

Kimble, J. and White, J. (1981). On the control of germ cell development in Caenorhabditis elegans. Dev. Biol. 81, 208-219.

Lin, H. and Spradling, A. (1993). Germline stem cell division and egg chamber development in transplanted Drosophila germaria. Dev. Biol. 159, 140-152.

Lindsley and Zimm (1992). The genome of Drosophila melanogaster. San Diego, California: Academic Press.

Lipshitz, H. D. and Kankel, D. R. (1985). Specificity of gene action during central nervous system development in Drosophila melanogaster: analysis of the lethal (1) optic ganglion reduced locus. Dev. Biol. 108, 56-77.

Meyerowitz, E. M. and Kankel, D. R. (1978). A genetic analysis of visual system development in Drosophila melanogaster. Dev. Biol. 62, 112-142.

Ogata, H., Taniguchi, S., Inaba, M., Sugawara, M., Ohta, Y., Inaba, K., Mori, K. J. and Ikehara, S. (1992). Separation of hematopoietic stem cells into two population and their characterization. Blood 80, 91-95.

Power, M. E. (1943). The effect of reduction in numbers of ommatidia upon the brain of Drosophila melanogaster. J. Exp. Zool. 94, 33-71.

Prokop, A. and Technau, G. M. (1991). The origin of postembryonic 


\section{S. Datta}

neuroblasts in the ventral nerve chord of Drosophila melanogaster. Development 111, 79-88

Prokop, A. and Technau, G. M. (1994). Normal function of the mushroom body defect gene of Drosophila is required for the regulation of the number and proliferation of neuroblasts. Dev. Biol. 161, 321-337.

Reynolds, B. A., Tetzlaff, W. and Weiss, S. (1992). A multipotent EGFresponsive striatal embryonic progenitor cell produces neurons and astrocytes. J. Neurosci. 12, 4565-4574.

Reynolds, B. A. and Weiss, S. (1992). Generation of neurons and astrocytes from isolated cells of the adult mammalian central nervous system. Science 255, 1707-1710.

Robbins, L. G. (1983). Maternal-zygotic lethal interactions in Drosophila melanogaster: zeste-white region single-cistron mutations. Genetics 103, 633-648.

Selleck, S. B., Gonzalez, C., Glover, D. M. and White, K. (1992). Regulation of G1-S transition in postembryonic neuronal precursors by axon ingrowth. Nature 355, 253-255.

Selleck, S. B. and Steller, H. (1991). The influence of retinal innervation on neurogenesis in the first optic ganglion of Drosophila. Neuron 6, 83-99.

Shannon, M. P., Kaufman, T. C., Shen, M. W. and Judd, B. H. (1972).

Lethality patterns and morphology of selected lethal and semi-lethal mutations in the zeste-white region of Drosophila melanogaster. Genetics 72, 615-638.

Spangrude, G. J. and Johnson, G. R. (1990). Resting and activated subsets of mouse multipotent hematopoietic stem cells. Proc. Natl. Acad. Sci. USA 87, 7433-7437.

Srour, E. F., Brandt, J. E., Briddell, R. A., Leemhuis, T., van Besien, K. and Hoffman, R. (1991). Human CD34+ HLA-DR- bone marrow cells contain progenitor cells capable of self-renewal, multilineage differentiation, and long-term in vitro hematopoiesis. Blood Cells 17, 287-295.

Truman, J. W. and Bate, M. (1988). Spatial and temporal patterns of neurogenesis in the central nervous system of Drosophila melanogaster. Dev. Biol. 125, 145-157.

Uchida, N. and Weissman, I. L. (1992). Searching for hematopoietic stem cells: Evidence that Thy-1.1 lo Lin- Sca+ cells are the only stem cells in C57BL/Ka Th-1.1 Bone marrow. J. Exp. Med. 175, 175-184.

White, K. and Kankel, D. R. (1978). Patterns of cell division and cell movement in the formation of the imaginal nervous system in Drosophila melanogaster. Dev. Biol. 65, 296-321.

(Accepted 19 December 1994) 